\title{
Effect of Radiation on Heat Transfer over an Exponentially Stretching Surface with MHD
}

\author{
M. Dakshinamoorthy ${ }^{1}$, P. Geetha ${ }^{2}$, M.B.K.Moorthy ${ }^{3}$ \\ ${ }^{I}$ (Department of Physics, Institute of Road and Transport Technology, Erode, India) \\ ${ }^{2}$ (Department of Mathematics, Bannari Amman of Institute of Technology, Sathyamangalam, India) \\ ${ }_{3}^{3}$ (Department of Mathematics, Institute of Road and Transport Technology, Erode, India)
}

\begin{abstract}
The effect of radiation on magnetohydrodynamic (MHD) boundary layer flow of a viscous fluid over an exponentially stretching sheet was examined. The governing partial differential equation are nondimensionalised and transformed into a system of nonlinear ordinary differential similarity equations, in a single independent variable $\eta$ and using RungeKutta Gill method with shooting technique. The velocity and temperature distributions are discussed numerically and presented through graphs. It was found that the local heat transfer rate at the surface decreases with increasing values of the magnetic and radiation parameters.
\end{abstract}

Keywords: Boundary layer, heat transfer, $M H D$, radiation, stretching sheet.

\section{Introduction}

The boundary layer flow on a continuous stretching sheet has attracted considerable attention during the last few decades due to its numerous applications in industrial manufacturing processes such as hot rolling, wire drawing, glass-fiber and paper production, drawing of plastic films, metal and polymer extrusion and metal spinning. Both the kinematics of stretching and the simultaneous heating or cooling during such processes has a decisive influence on the quality of the final products (Magyari \& Keller 1999). Magyari et al (2001) reported analytical and computational solutions when the surface moves with rapidly decreasing velocity using self similarity method.

Magnetohydrodynamics flows have applications in meteorology, solar physics, cosmic fluid dynamics, astrophysics, geophysics and in the motion of earth's core. In addition from the technological point of view, MHD free convection flows have significant applications in the field of stellar and planetary magneto spheres, aeronautical plasma flows, chemical engineering and electronics.

With general power function form for stretching velocity of the wall, the same problem has been analyzed with surface suction injection by Ali and Al-Yousef (2002). Magyari and Keller (1999) investigated the steady boundary layers on an exponentially stretching continuous surface with an exponential temperature distribution. Partha et al. (2005) studied the effect of viscous dissipation on the mixed convection heat transfer from an exponentially stretching surface. Recently, Sajid and Hayat (2008) extended this problem by investigating the radiation effects on the flow over an exponentially stretching sheet, and solved the problem analytically using the homotopy analysis method. The numerical solution for the same problem was then given by Bidin and Nazar (2009). The study of magnetohydrodynamic has important applications, and may be used to deal with problems such as cooling of nuclear reactors by liquid sodium and induction flow meter, which depends on the potential difference in the fluid in the direction perpendicular to the motion and to the magnetic field Ganesan \& Palani (2004). At high operating temperature, radiation effect can be quite significant. Many processes in engineering areas occur at high temperatures and knowledge of radiation heat transfer becomes very important for the design of pertinent equipment (Seddeek 2002).

The aim of the present paper is to study the effect of radiation on heat transfer over an exponentially stretching surface with MHD which has not been considered before.

\section{Problem Formulation}

A steady two dimensional flow of an incompressible viscous and electrically conducting fluid caused by a stretching sheet, which is placed in a quiescent ambient fluid of uniform temperature $T_{\infty}$, as shown in Fig.1. A variable magnetic field $\mathrm{B}(\mathrm{x})$ is applied normal to the sheet and that the induced magnetic field is neglected, which is justified for MHD flow at small magnetic Reynolds number. Under the usual boundary layer approximations, the flow and heat transfer with the radiation effects are governed by the following equations:

$$
\begin{aligned}
& \frac{\partial u}{\partial x}+\frac{\partial v}{\partial y}=0 \\
& u \frac{\partial u}{\partial x}+v \frac{\partial u}{\partial y}=v \frac{\partial^{2} u}{\partial y^{2}}-\frac{\sigma B^{2} u}{\rho}
\end{aligned}
$$


$u \frac{\partial T}{\partial x}+v \frac{\partial T}{\partial y}=\frac{k}{\rho c_{p}} \frac{\partial^{2} T}{\partial y^{2}}-\frac{1}{\rho c_{p}} \frac{\partial q_{r}}{\partial y}$

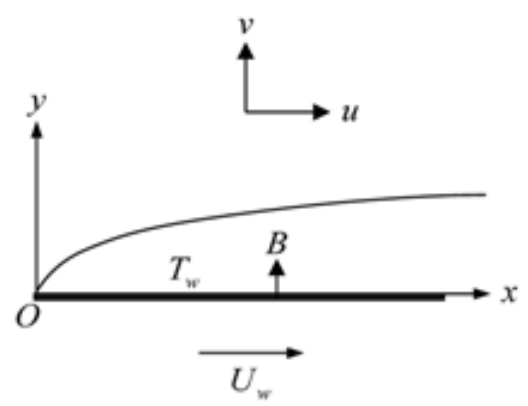

Fig. 1. Physical model and Coordinate system

where $u$ and $v$ are the velocities in the $x$ and $y$ - directions, respectively, $\rho$ is the fluid density, $v$ the kinematics viscosity, $\mathrm{k}$ the thermal conductivity, $\mathrm{c}_{\mathrm{p}}$ the specific heat, $\mathrm{T}$ the fluid temperature in the boundary layer and $\mathrm{q}_{\mathrm{r}}$ is the radiative heat flux. The boundary conditions are given by

$u=U_{w}=U_{0} e^{x / L}, v=0$

$T=T_{w}=T_{\infty}+T_{0} e^{x / 2 L} \quad$ at $y=0$

$u \rightarrow 0, T \rightarrow T_{\infty}$ as $y \rightarrow \infty$

where $\mathrm{U}_{0}$ is the reference velocity, $\mathrm{T}_{0}$ the reference temperature and $\mathrm{L}$ is the reference length. Most of the effort in understanding fluid radiation is devoted to the derivation of reasonable simplifications (Aboeldahab \& El Gendy 2002).

For an optically thick gas, the gas self-absorption rises and the situation become difficult. However, the problem can be simplified by using the Rosseland approximation which simplifies the radiative heat flux as:

$q_{r}=-\frac{4 \sigma^{*}}{3 k^{*}} \frac{\partial T^{4}}{\partial y}$

where $\sigma^{*}$ and $\mathrm{k}^{*}$ are the Stefan-Boltzmann constant and the mean absorption coefficient, respectively. This approximation is valid at points optically far from the boundary surface, and it is good only for intensive absorption, which is for an optically thick boundary layer (Bataller 2008). It is assumed that the temperature differences within the flow such that the term $\mathrm{T}^{4}$ may be expressed as a linear function of temperature. Hence, expanding $\mathrm{T}^{4}$ in a Taylor series about $\mathrm{T}_{\infty}$ and neglecting higher-order terms gives:

$T^{4}=4 T_{\infty}^{3} T-3 T_{\infty}^{4}$

Using Eqs. (5) and (6), Eq. (3) reduces to:

$u \frac{\partial T}{\partial x}+v \frac{\partial T}{\partial y}=\frac{k}{\rho c_{p}} \frac{\partial^{2} T}{\partial y^{2}}+\frac{16 \sigma^{*} T_{\infty}^{3}}{3 \rho c_{p} k^{*}} \frac{\partial^{2} T}{\partial y^{2}}$

To obtain similarity solutions, it is assumed that the magnetic field $\mathrm{B}(\mathrm{x})$ is of the form $\mathrm{B}=\mathrm{B}_{0} \mathrm{e}^{\mathrm{x} / 2 \mathrm{~L}}$

where $\mathrm{B}_{0}$ is the constant magnetic field.

The continuity equation (1) is satisfied by introducing a stream function $\psi$ such that

$u=\frac{\partial \psi}{\partial y}$ and $v=-\frac{\partial \psi}{\partial x}$

The momentum and energy equations can be transformed into the corresponding ordinary differential equations by the following transformation (Sajid \& Hayat 2008):

$$
\begin{aligned}
& u=U_{0} e^{x / L} f^{\prime}(\eta), \quad v=-\left(\frac{v U_{0}}{2 L}\right)^{1 / 2} e^{x / 2 L}\left(f(\eta)+\eta f^{\prime}(\eta)\right) \\
& T=T_{\infty}+T_{0} e^{x / 2 L} \theta(\eta), \quad \eta=\left(\frac{U_{0}}{2 v L}\right)^{1 / 2} e^{x / 2 L} y
\end{aligned}
$$


where $\eta$ is the similarity variable, $f(\eta)$ is the dimensionless stream function, $\theta(\eta)$ is the dimensionless temperature and primes denote differentiation with respect to $\eta$. The transformed ordinary differential equations are:

$$
\begin{aligned}
& f^{\prime \prime \prime}+f f^{\prime \prime}-2 f^{\prime 2}-M f^{\prime}=0 \\
& \left(1+\frac{4}{3} K\right) \theta^{\prime \prime}+\operatorname{Pr}\left(f \theta^{\prime}-f^{\prime} \theta\right)=0
\end{aligned}
$$

in which $M=\frac{2 \sigma B_{0}^{2} L}{\rho U_{0}}$ is the magnetic parameter, $K=\frac{4 \sigma^{*} T_{\infty}^{3}}{k^{*} k}$ the radiation parameter and $\operatorname{Pr}=\frac{\rho v c_{p}}{k}$ is the Prandtl number. The transformed boundary conditions are:

$$
\begin{array}{ll}
f(0)=0, \quad f^{\prime}(0)=1, \quad \theta(0)=1 & \text { at } \eta=0 \\
f^{\prime}(\infty) \rightarrow 0, \theta(\infty) \rightarrow 0 & \text { as } \eta \rightarrow \infty
\end{array}
$$

The skin-friction coefficient and the local Nusselt number which represents the wall shear stress and the heat transfer rate at the surface, respectively are important physical parameters for this type of boundary layer flow.

\section{Solution Of The Problem}

The set of coupled non-linear governing boundary layer equations (11) and (12) together with the boundary conditions (13) are solved numerically by using Runge-Kutta Gill method along with the shooting technique. First of all, higher order non-linear differential equations (11) and (12) are converted into simultaneous linear differential equations of first order and they are further transformed into initial value problem by applying the shooting technique. The resultant initial value problem is solved by employing RungeKutta Gill method. The step size $\Delta \eta=0.05$ is used to obtain the numerical solution with five decimal place accuracy as the criterion of convergence. From the process of numerical computation, the local Nusselt number, which is proportional to - $\theta^{\prime}(0)$ is also sorted out and their numerical values are presented in a tabular form.

\section{Figures and Tables}

Table 1. Values of $\theta^{\prime}(0)$ for different values of $\mathrm{K}, \mathrm{M}$ and $\operatorname{Pr}$

\begin{tabular}{ccclccc}
\hline $\mathrm{K}$ & $\mathrm{M}$ & Pr & $\begin{array}{l}\text { Magyari and Keller } \\
(1999)\end{array}$ & El-Aziz (2009) & $\begin{array}{c}\text { Bidin and Nazar } \\
(2009)\end{array}$ & Present results \\
\hline 0 & 0 & 1 & -0.954782 & -0.954785 & -0.9548 & -0.9548 \\
& & 2 & & & -1.4714 & -1.4715 \\
& & 3 & -1.869075 & -1.869074 & -1.8691 & -1.8691 \\
& & 5 & -2.500135 & -2.500132 & & -2.5001 \\
& & 10 & -3.660379 & -3.660372 & & -3.6603 \\
& 1 & 1 & & & -0.5315 & -0.8613 \\
& 0 & & & & -0.4504 \\
\hline
\end{tabular}

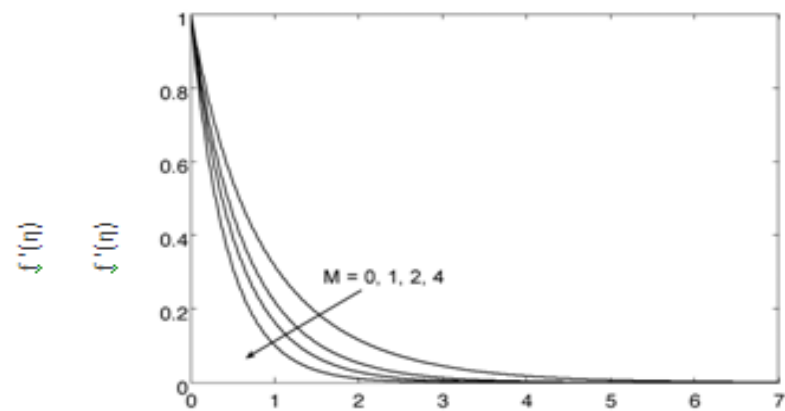

$\eta$

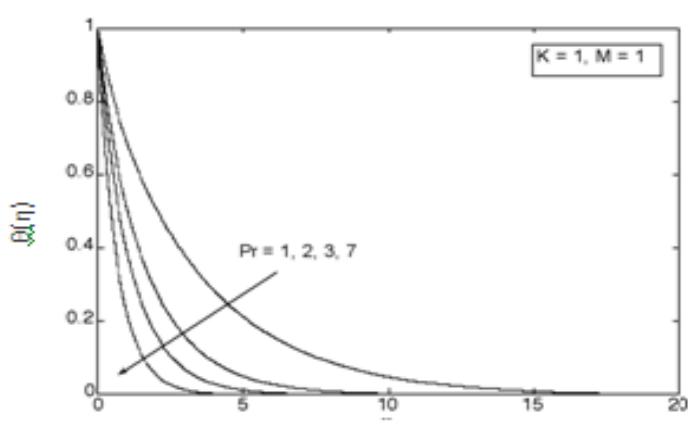

$\eta$

Fig. 3. Temperature profile for different values of $\operatorname{Pr}$ 


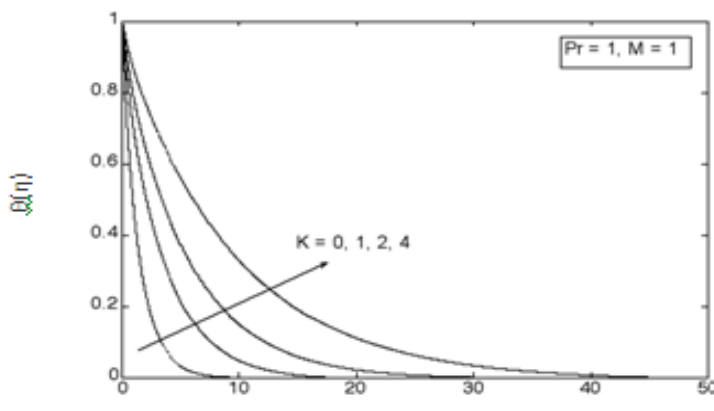

$\eta$

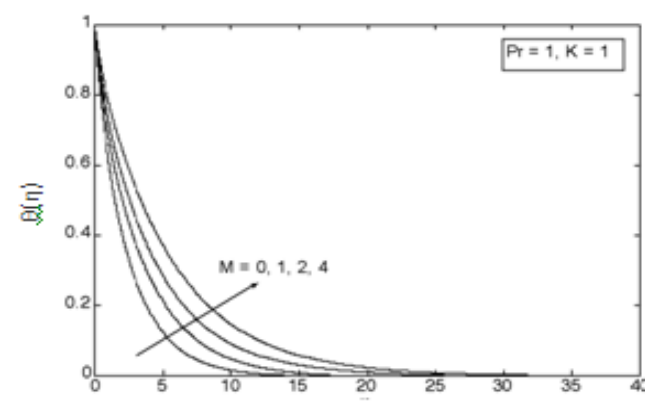

$\eta$

Fig. 4 Temperature profiles for different values of $\mathrm{K}$

Fig. 5 Temperature profile for different values of M

\section{Results and Discussion}

Table 1 shows numerical values of magnetic field parameter $\mathrm{M}$, radiation parameter $\mathrm{K}$ and Prandtl number Pr effects on - $\theta^{\prime}(0)$. Comparison with the existing results from the literature gives a favourable agreement, as presented in Table 1.

The profiles for velocity and temperature are shown in Fig.2-5 respectively with various values of the parameters. The velocity profiles for different values of the magnetic parameter M presented in Fig. 2 show that the rate of transport is considerably reduced with the increase of M. It clearly indicates that the transverse magnetic field opposes the transport phenomena. This is because the variation of $\mathrm{M}$ leads to the variation of the Lorentz force due to the magnetic field, and the Lorentz force produces more resistance to the transport phenomena. We note that the Prandtl number Pr and the radiation parameter $\mathrm{K}$ have no influence on the flow field, which is clear from Equation (11).The velocity gradient at the surface $f$ " $(0)$ which represents the surface shear stress increases with increasing $\mathrm{M}$. Thus, the magnetic parameter $\mathrm{M}$ acts as a controlling parameter to control the surface shear stress.

The temperature profiles for different values of $\operatorname{Pr}, \mathrm{K}$ and $\mathrm{M}$ with other parameters are fixed to unity are presented in Figs. 3, 4 and 5, respectively. Figs. 2 to 5 show that the far field boundary conditions are satisfied asymptotically, thus supporting the accuracy of the numerical results obtained. It is evident from Figs. 3 to 5 that the thermal boundary layer thickness increases as $\mathrm{M}$ and $\mathrm{K}$ increase but opposite trends are observed for increasing values of Pr. This results in decreasing manner of the local Nusselt number $-\theta^{\prime}(0)$, which represents the heat transfer rate at the surface, with increasing $\mathrm{M}$ and $\mathrm{K}$ but opposite trends are observed for increasing values of Pr. This is because, when Pr increases, the thermal diffusivity decreases and thus the heat is diffused away from the heated surface more slowly and in consequence increase the temperature gradient at the surface

\section{Conclusion}

The effect of radiation on heat transfer over an exponentially stretching surface with MHD was investigated. The numerical results obtained agreed very well with previously reported cases available in the literature. It was found that the surface shear stress increases with the magnetic parameter $\mathrm{M}$, while the heat transfer rate increases with Prandtl number $\mathrm{Pr}$, but decreases with both magnetic parameter $\mathrm{M}$ and radiation parameter K.

\section{Acknowledgements}

The authors wish to thank the Chairman, Trustee, Chief Executive, Principal, Bannari Amman Institute of Technology for their support to do this work. The authors wish to thank the Director IRT Chennai and the Principal IRTT, Erode for their generous help.

References

[1] E. Magyari, and B. Keller, Heat and mass transfer in the boundary layers on an exponentially stretching continuous surface. J. Phys. D: Appl. Phys. 32: 1999, 577-585.

[2] E. Magyari, M.E.Ali and B. Keller B, Heat and mass transfer characteristics of the self similar boundary layer flows induced by continuous surface stretched with rapidly decreasing velocities. Heat Mass Transfer 38: 2001, 65-74.

[3] M. Ali, and F. Al-Yousef, Laminar mixed convection boundary layers induced by a linearly stretching permeable surface. Int J Heat Mass Transfer 45: 2002,4241-4250.

[4] M.K. Partha, P.V.S.N. Murthy, and G.P. Rajasekhar, Effect of viscous dissipation on the mixed convection heat transfer from an exponentially stretching surface. Heat Mass Transfer 41:2005, 360-366.

[5] M. Sajid, and T. Hayat, Influence of thermal radiation on the boundary layer flow due to an exponentially stretching sheet. Int. Comm. Heat Mass Transfer 35: 2008, 347-356. 
[6] B. Bidin, and R. Nazar, Numerical solution of the boundary layer flow over an exponentially stretching sheet with thermal radiation. Euro J. Sci. Res. 33(4): 2009, 710-717.

[7] P. Ganesan, and G. Palani, Finite difference analysis of unsteady natural convection MHD past an inclined plate with variable surface heat and mass flux. Int. J. Heat Mass Transfer 47: 2004, 4449-4457.

[8] M.A.Seddeek, Effects of radiation and variable viscosity on a MHD free convection flow past a semi-infinite flat plate with an aligned magnetic field in the case of unsteady flow. Int. J. Heat Mass Transfer 45: 2002, 931-935.

[9] E. M. Aboeldahab, and M.S. El Gendy, Radiation effect on MHD free convective flow of a gas past a semi-infinite vertical plate with variable thermophysical properties for high temperature differences. Can. J. Phys. 80: 2002, 1609-1619.

[10] R.C. Bataller, Similarity solutions for boundary layer flow and heat transfer of a FENE-P fluid with thermal radiation. Phys. Lett. A 372: $2008,2431-2439$ 